inium (specific surface $1,600 \mathrm{~cm} .{ }^{2} / \mathrm{gm}$.) of density 0.75 gra. $/ \mathrm{cm}^{3}$ and cartridge diameter $\frac{7}{8}$ in. The velocity of detonation was measured photographically.

For the T.N.T. the velocity of detonation in air was $1,380 \pm 20 \mathrm{~m} . / \mathrm{s}$., and in argon $1,580 \pm 15 \mathrm{~m} . / \mathrm{s}$. For the ammonium nitrate composition, the velocities were $2,850 \pm 30 \mathrm{~m} . / \mathrm{s}$., and $3,100 \pm 30 \mathrm{~m} . / \mathrm{s}$., respectively, the limits quoted being standard deviations. There is clearly a significant increase in velocity, which further confirms the view that the mechanism of propagation of detonation involves heating of the gas spaces by the shock front.

T. L. CotTrelel

J. G. GrBB

Research Department,

Nobel Division,

Imperial Chemical Industries, Ltd., Stevenston, Ayrshire. June 26.

2 Taylor, J., "Detnnation in Condensed Explosives", Chap. xi (Oxford, 1952).

Paterson, S., Fifth Symposium (International) on Combustion, New York, 672 (1955).

\section{Effect of Lightning Discharges on Magnetographs}

Chaubal, Deshmukh and Malurkar ${ }^{1}$ recently reported an instance of "minor oscillations" recorded particularly by the La Cour $H$ and $D$ magnetographs of the Alibag Observatory during a thunderstorm which passed over Alibag on June 2, 1955. They were interested to know if similar observations were available from other observatories.

Since 1952, two complete sets of photographically recording magnetographs, one Watson and the other La Cour, have been functioning regularly in the Magnetic Section of the Kodaikanal Observatory. During the past four years or more, in the thunderstorm seasons, I have often noticed in the traces of these magnetographs oscillations of precisely the same character as reported by Mr. S. L. Malurkar and his co-workers. I have always thought them to be caused by lightning discharges. However, I have now examined all our La Cour and Watson records from 1952 to date in order to check the correctness of the impression that these characteristic oscillations are of rather common occurrence and are indeed associated with thunderstorms. In our records there are some dozens of instances of such oscillations which correspond with thunder and lightning reported by our weather observer. There are also some instances in which similar, though usually lessmarked, oscillations are noticeable, but no corresponding thunderstorms are recorded in the weather observer's diary. The cause of these latter oscillations is obscure ; perhaps, during the normal watch hours the weather observer has not noticed the somewhat distant or minor lightning discharges, and presumably has not reported all the thunderstorms that may have occurred late in the night. It is also not impossible that some of the very feeble oscillations are due to the momentary, but heavy, direct current which the battery supplies to the self-starter of a motor-car engine starting up rather close to the magnetic observatory. However, in all cases in which there is correspondence between the magnetic oscillations recorded and the lightning reported there can be little doubt but that it is the short-duration electric currents accompanying lightning strokes which are responsible for the perturbations recorded by the magnetographs.

The variometer room at Kodaikanal is a very substantially built, thermally well-insulated chamber with a thick roof and heavy double walls of stone masonry. Both the Watson and La Cour variometers are installed on piers supported by the masonry floor in exactly the same manner. The sensitivity (taken to mean the ability to respond fully to a variation of duration comparable or greater than the period of the instrument) of the Watson $H F$ instrument is considerably higher than that of the corresponding La Cour instrument. It does not seem that the method of mounting of a magnetograph has much to do with its capacity for recording the oscillations caused by impulsive lightning discharges.

From Kodaikanal records it is clear that often the magnetic oscillations due to lightning strokes occur simultaneously in all the three traces $(H, D$ and $V)$ of the La Cour variometers, although the amplitudes are different in the three components, evidently because the corresponding intensities are also different. We have many instances in which the Watson instruments (especially $H$ and $D$, the performance of the $V F$ instrument being often unsatisfactory) have also recorded such oscillations during thunderstorms; but these oscillations have smaller amplitudes than those recorded by the La Cour variometers, and are very much damped. These differences are evidently attributable to the fact that compared to the La Cour variometers the Watson magnetographs have considerably greater inertia and higher damping.

I am indebted to Mr. R. V. Subramanian for assistance in examining the magnetic records.

Astrophysical Observatory,

A. K. DAS

Kodaikanal.

May 30. 1 Chaubal, A. S., Deshmukh, D. K., and Malurkar, S. L., Nature, 176,
876 (1955).

\section{Vowel Sounds and Perceptual Constancy}

IT has been known for some time that vowels are distinguished from one another mainly by the frequencies of the formants or regions of the auditory spectrum in which there is a comparatively large amount of energy. Recently, the hypothesis has been put forward by $\mathrm{J}_{00 \mathrm{~s}^{1}}$ that when a listener identifies the vowel in a particular word he does so not by considering the absolute value of the lowest two formant frequencies, as was previously thought, but by assessing the frequencies of these formants in relation to the formant frequencies of other vowels which he has heard pronounced by that speaker. On this hypothesis, the identification of a particular sound depends on the acoustic structure of the neighbouring sounds.

A similar process is known to occur in the perception of other kinds of sensory data. Thus in visual experiments the same response, 'white', may be given to a piece of paper in sunlight and in an artificially lighted room, despite the wide differences in the spectra of the light.reflected from the paper: the other neighbouring stimuli compensate for the differences ${ }^{2}$.

It has recently become possible to demonstrate the auditory phenomenon with the aid of synthetic speech, which can now be precisely controlled and 